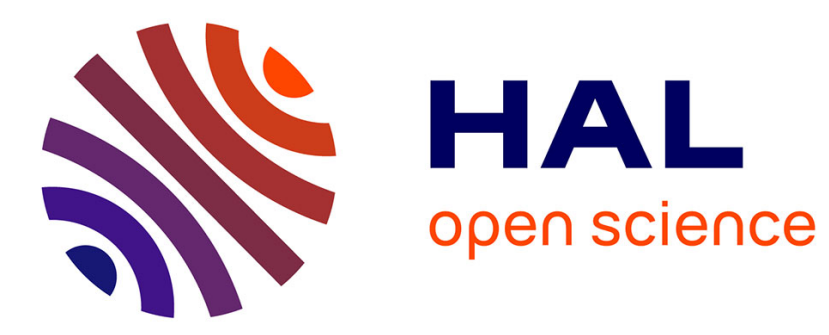

\title{
Se situer parmi les pairs : des groupes affinitaires aux conflits. L'étude d'interactions dans une cour de récréation
}

Émilie Salaméro

\section{- To cite this version:}

Émilie Salaméro. Se situer parmi les pairs : des groupes affinitaires aux conflits. L'étude d'interactions dans une cour de récréation. Education et Sociétés : Revue internationale de sociologie de l'éducation, 2016, 38 (2), pp.157-172. 10.3917/es.038.0157 . hal-03388535

\section{HAL Id: hal-03388535 \\ https://hal.science/hal-03388535}

Submitted on 20 Oct 2021

HAL is a multi-disciplinary open access archive for the deposit and dissemination of scientific research documents, whether they are published or not. The documents may come from teaching and research institutions in France or abroad, or from public or private research centers.
L'archive ouverte pluridisciplinaire HAL, est destinée au dépôt et à la diffusion de documents scientifiques de niveau recherche, publiés ou non, émanant des établissements d'enseignement et de recherche français ou étrangers, des laboratoires publics ou privés. 
Salaméro Émilie,

Maître de conférences, université de Poitiers.

Membre du CEREGE (EA 1722), associée à l'équipe CreSco (EA 7419)

emilie.salamero@univ-poitiers.fr

Titre : Positionnement parmi les pairs: des groupes affinitaires aux conflits. L'étude d'interactions dans une cour de récréation

\section{Résumé :}

Les enfants sont souvent appréhendés comme un groupe social homogène et leurs conflits «ordinaires» sont peu étudiés. Une enquête ethnographique menée pendant un an dans une école ariégeoise auprès d'une classe de CM2 permet de porter un regard plus attentif aux conflits entre filles et à leur rôle au sein des pairs, en tenant compte de la structure des relations sociales. Dans ce travail, les modalités de conduite des conflits sont reliées aux modes de socialisation des enfants et à leurs ressources sociales, analysées à partir des pratiques corporelles (activités ludiques et apparence corporelle) développées en cour de récréation. Cette étude met plus particulièrement au jour le rôle des dispositions agonistiques au sein des rapports sociaux enfantins et dans le processus de hiérarchisation des filles.

Mots clés : école, socialisation entre pairs, groupes affinitaires, pratiques corporelles, hiérarchisation. 
Les sciences sociales s'intéressent depuis peu à l'enfance et ont renouvelé leur regard sur cet âge de la vie (Rayou, 1999; Sirota, 1988). Dans cette perspective, une attention nouvelle a été accordée aux interactions entre pairs dans la cour de récréation. Au sein de l'enceinte scolaire, elle constitue un espace privilégié pour l'étude des rapports horizontaux entre pairs (Delalande, 2001), dont l'influence se renforce à la préadolescence (Octobre, Jauneau, 2008).

Lors d'une enquête ethnographique menée auprès d'une classe de CM2, les conflits enfantins ont ponctué les interactions quotidiennes et apparaissent en ce sens " ordinaires ». Selon certaines approches, ces conflits sont liés au processus d'incorporation des normes dominantes, qu'elles soient imposées par les adultes (Geay, 2003) ou définies comme acceptables par les pairs (Carra, 2008). D’autres perçoivent les inimitiés enfantines comme moyen d'instaurer précocement des distances sociales (Lignier, Pagis, 2014). Les réseaux de sociabilité sont en effet traversés par des enjeux de positionnement (Carra, 2008) et rapports de pouvoir (Geay, 2000). La compréhension des conflits passe par leur objectivation (Rui, 2011) et la popularité des élèves, liée à leurs ressources et propriétés sociales (Adler, Kless, Adler, 1992), peut en constituer un indicateur.

Parmi la classe observée, majoritairement composée de filles, ce sont plus précisément les conflits féminins, inscrits dans la durée et commentés par les protagonistes, qui sont apparus plus accessibles que ceux des garçons, localisés et réglés sur le registre de l'opposition brutale (Brougère, 1999). Ce constat nous amène à nous interroger sur le rôle des modes de socialisation dans le développement des conflits enfantins. Chez les filles, ces conflits « ordinaires » semblent tenir une place importante dans la construction identitaire, du fait de la prégnance de la norme d'exclusivité qui les lie à leur groupe affinitaire (Monnot, 2013) et à leur mode de socialisation particulièrement discursif (Octobre, 2003). Si les groupes de pairs participent au processus d'incorporation de la féminité (Monnot, 2013), il apparaît aujourd'hui nécessaire de tenir compte de la variabilité des formes de féminité et aux instances de socialisation qui participent à cette différenciation. Dans cette perspective, nous nous proposons d'observer la variété des modes de conduite des conflits chez les filles.

Dans la continuité des travaux qui s'intéressent à la socialisation entre pairs dans l'espace scolaire (Geay, 2000 ; Comoretto, 2010 ; Mardon, 2010), la variabilité des conflits féminins à l'école est reliée aux modes de socialisation des enfants, à leurs ressources sociales et dispositions, particulièrement observables dans certaines pratiques enfantines. En effet, le corps est ici envisagé comme un capital distinctif total (Darmon, 2008). Étudier les pratiques, usages et catégories de perception du corps chez les filles constitue un moyen pour 
appréhender les modes de structuration de cette micro société que constitue la cour de récréation.

L'analyse des conflits ne pouvant se passer d'un examen attentif de la structure des parties en présence (Simmel, 2003) et du cadre social dans lesquels ils se développent (Lignier, Pagis, 2012), la distribution des filles autour de groupes affinitaires différenciés sera dans un premier temps présentée, ainsi que leurs pratiques ludiques, dispositions sportives et scolaires. Dans un second temps, l'exposé sera nourri par deux conflits impliquant des groupes affinitaires distincts, qui nous permettront d'observer comment les dispositions agonistiques participent, au sein du contexte étudié, au phénomène de hiérarchisation des filles.

\section{Appréhender les rapports sociaux dans la cour de récréation via une enquête ethnographique}

Parmi les données récoltées (200 heures) tout au long d'une année scolaire (2011-2012) auprès d'enfants de CM2 scolarisés dans une école publique de 176 élèves d'une commune ariégeoise rurale d'environ 3000 habitants $^{\mathrm{i}}$, cet article s'appuie essentiellement sur les observations menées dans la cour de récréation. L'école et la classe suivie, accueillaient une population socialement mixte, avec une prédominance des classes moyennes et populaires, à l'instar de la commune (données de l'INSEE, 2013).

Même s'il n'est pas aisé de s'immiscer, en tant qu'enquêteur adulte, dans les jeux et relations interpersonnelles d'enfants âgés de 10 à 11 ans, notre présence régulière dans l'enceinte de l'école et une « observation discrète » (Gayet, 2006), ont permis d'établir une relation de confiance et de faire oublier aux enfants, à certains moments, notre rôle. La dynamique des interactions a été ainsi replacée dans le contexte d'enquête, ce qui constitue un apport indéniable de l'approche socio-ethnographique ; les entretiens formels classiques étant souvent inadaptés à cette population spécifique (Détrez, 2014). Cette méthodologie a été complétée par la réalisation d'entretiens auprès des enseignants de la classe, rendus indispensables par l'absence de certaines données dans les dossiers scolaires (résultats moyens et informations familiales), certains enfants (13) et un nombre limité de familles (6).

\section{Gestion des temps libres à l'école :}

En dehors de la coupure méridienne de deux heures, deux récréations d'une durée de 20 minutes environ ont constitué le support d'observation des interactions entre enfants, suivis également à la cantine et en classe. Le temps méridien est encadré par des animateurs du 
centre de loisirs qui proposent aux enfants, s'ils le souhaitent, des activités avant leur repas (jeux de société, activités manuelles, tournois, etc.). Le temps de récréation est pris en charge par l'équipe enseignante et les élèves y organisent seuls leur temps libre, avec, sur demande, de petits matériels fournis par l'école : ballons, diabolos, cerceaux, élastiques, etc. Certains effets personnels sont aussi autorisés dans le règlement de l'école : billes, magazines, etc. La cour des CM, composée de terrains de football/basketball et d'un préau avec toilettes mixtes, est séparée de celle des CP-CE.

L'appartenance à un groupe de sexe constitue un élément central de la construction identitaire à l'enfance et à l'adolescence. Toutefois, le suivi des élèves dans les différents espaces de l'école a permis de cerner, parmi les 15 filles de la classe (sur 26), deux grands groupes affinitaires, deux duos et une élève en marge de la classe (cf. graphique 1) et, pour chacun de ces groupes, les pratiques récréatives, comportements en cours d'Éducation Physique et Sportive et apparence vestimentaire. En effet, celle-ci joue un rôle important dans la popularité des filles (Adler, Kless, Adler, 1992) et dans la différenciation des adolescents (Mardon, 2010).

Graphique 1 : les groupes affinitaires au sein de la classe 


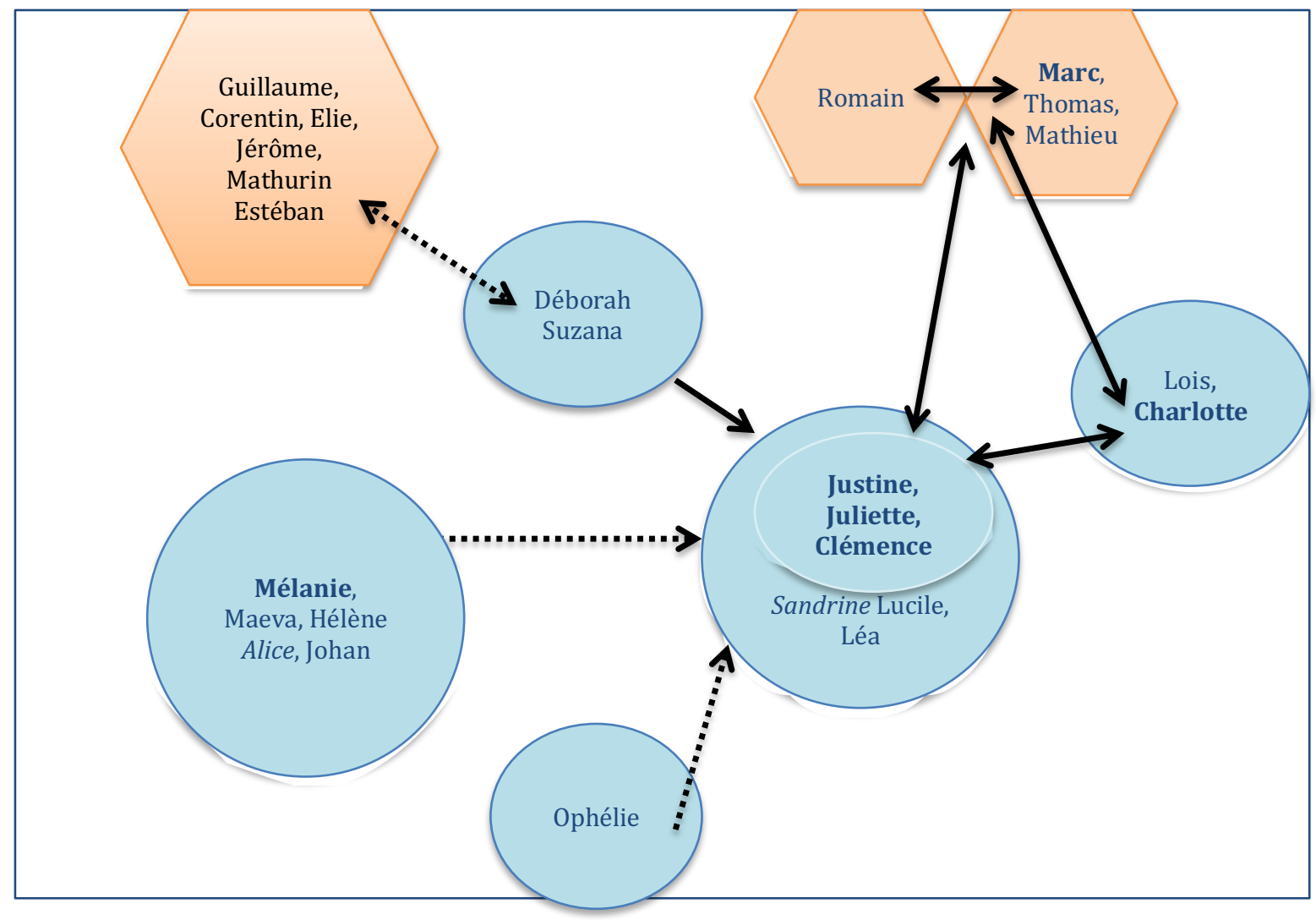

\section{Légende:}

- prénom en italique : membre rejeté du groupe en CM2

- prénom en gras : leader et noyau du groupe affinitaire

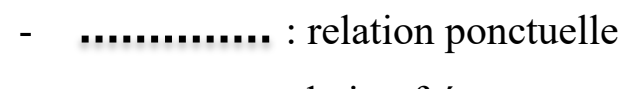

- $\longrightarrow$ : relation fréquente

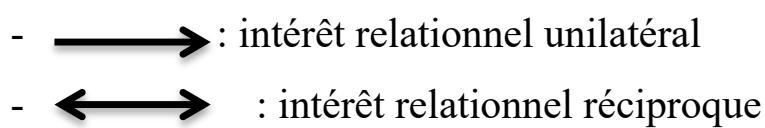

Un premier groupe est composé de quatre à six filles selon les périodes (Justine, Juliette, Clémence, Sandrine, Lucile et Léa). Entre les CM1 et CM2, quatre enfants ont été rejetés par ses membres, signe que ce groupe est convoité et contraint à une redéfinition régulière de ses frontières. Dans la cour de récréation, ses activités sont diverses ainsi que les espaces occupés. Ses membres investissent tout d'abord des activités traditionnellement investies par les filles : calmes (histoires qui font peur, coloriage de cahiers de stylisme, jeux de carte, etc.) et partagées avec d'autres filles ; gymniques/dansées (mouvements de rock and roll, roues sur l'herbe), ou prolongeant les projets de classe (exposés, fabrication de décors pour un spectacle, etc.).

Justine fait des roues dans le carré d'herbe situé près des bureaux du centre de loisirs en début de temps méridien. Pendant ce temps, Clémence me demande si elle, Justine et Juliette peuvent répondre aux questions de l'entretien «vite fait», parce qu'elles préparent un cahier personnalisé pour Malo (ancien camarade qui vient de déménager) avec « tout ce qu'il aime ». Clémence a apporté un classeur 
où elle rassemble des photographies de star découpées et des dessins faits par d'autres camarades. Elle veut compiler le tout dans un cahier puis lui envoyer. (journal de terrain du 10 octobre 2011)

Par ailleurs, elles s'adonnent à des jeux perçus comme plus masculins avec les garçons les plus turbulents de la classe (Marc, Romain, Mathieu et Thomas) : jeux au ballon (tennisballon, passe à dix), babyfoot, jeux d'attrape, etc. Dans ces derniers, elles mobilisent des registres d'interaction proches de ceux observés chez ces mêmes garçons, notamment pour répondre à leur provocation :

Alors que Justine observe le jeu de cartes proposé par Mélanie sur le rebord de la fenêtre de la classe, Romain passe à proximité d'elle et lui donne une grosse claque sur le bras. Justine se met à le poursuivre dans la cour, comme pour laver cet affront. Elle est rapidement aidée de Léa, Charlotte et Johan. D'autres élèves se joignent progressivement à eux, prenant soit partie pour Justine (Clémence) soit pour Romain (Marc, Thomas, Mathieu). (Journal de terrain du 14 mai 2012)

Les filles de ce groupe sont les plus sensibles aux codes vestimentaires prisés par les adolescents : elles portent de nombreuses marques (Teddy Smith, Bensimon, Converses, Levis, Kaporals, etc.), tenues et accessoires à la mode du moment (short en jean sur legging, bottines en aspect cuir avec une grosse boucle sur le côté). Elles accordent aussi une attention à leur coupe et/ou coiffure (petite barrette qui tient les cheveux de devant, dégradé long ou mi long).

Juliette se recoiffe à table, elle demande à Justine si sa «coiffure va » (journal de terrain du 3 octobre 2011)

A ce look féminin à la mode, s'ajoute un goût pour les marques sportwear et de glisse (Roxy, O’Neil, Nike, Adidas, Oxbow) portées les jours de sport scolaire. D'ailleurs, ces filles sont les plus sportives, que ce soit dans la cour de récréation, de par leurs activités extra scolaires ou en cours d'Éducation Physique et Sportive :

$\mathrm{Au}$ début du match de hockey entre les équipes de Juliette et Justine, toutes deux donnent des consignes à leur équipe. Juliette livre des éléments stratégiques à Johan et Alice. Justine dit à Romain « fais comme tout à l'heure, va au centre » : elle compte lui faire une passe directe. (Journal de terrain du 5 janvier 2012)

Elles mobilisent ainsi des registres récréatifs diversifiés, avec une certaine appétence pour les jeux d'opposition et codes de la culture préadolescente, tout en respectant les règles scolaires. Elles sont d'ailleurs considérées comme de « bonnes » élèves par leur enseignant et 
camarades. Même en dehors de la classe, les enfants restent soumis aux normes éducatives de type scolaire (Roucous, 2007) et s'appuient sur les classements institutionnels (Lignier et al, 2012), d'autant plus chez les filles (Adler, Kless et Adler, 1992).

Un deuxième groupe affinitaire numériquement important, est composé de quatre filles et d'un garçon (Mélanie, Hélène, Johan, Maeva et Alice). Accueillant deux puis trois membres rejetés (Johan et Hélène en CM1 ; Sandrine en CM2) par le groupe de Justine ainsi que des nouvelles dans la classe (Alice, Mélanie). Elles apprécient particulièrement des jeux qui nous pourrions qualifier d'enfantins (Geay, 2000), dans le sens où ils sont traditionnellement investis par des élèves plus jeunes et non par les CM2 : corde à sauter, chat perché, jeux de mains, etc.

A la récréation de l'après-midi et dès la sortie de la classe, Sandrine rejoint le groupe d'Alice, Hélène, Mélanie et Johan. Sous le préau, ils s'amusent à se faire tourner (l'un d'eux tient un autre par la main et le fait tourner autour de lui le plus rapidement possible jusqu'à ce qu'il lâche et tente de récupérer son équilibre), puis à faire des jeux de mains deux à deux. (Journal de terrain du 5 janvier 2012)

Plus à l'écart du reste de leurs camarades, notamment des garçons, elles observent fréquemment les premières dans leurs activités récréatives et partagent parfois avec elles des activités calmes. Mais lorsqu'une activité occupe l'ensemble de leur classe d'âge, comme les parties de bille en début d'année, elles optent pour des activités annexes, limitant leur appropriation de compétences spécifiques (Augustins, 1988).

Mélanie essaie de récupérer des billes tombées sous la grille d'évacuation des eaux avec une règle en plastique et de la pâte à fixe ; elle est entourée de plusieurs enfants qui la regardent faire (Ophélie, Thomas, Alice, Hélène et Maeva). Ophélie me montre son pot de billes cabossées ; Mélanie et ses copines n'en ont pas amenées. (journal de terrain du 3 octobre 2011)

$\mathrm{Au}$ niveau vestimentaire, elles se montrent plus distantes des codes de l'apparence corporelle prisée par les pré adolescentes : serre tête épais dans les cheveux, cheveux longs ou mi-longs sans coupe spécifique, absence de marques prisées, vêtements avec des personnages enfantins (Hello Kitty), tissus à pois ou à rayure, etc. L'accès aux jeux sportifs leur est difficile, si bien qu'elles sont stimulées par les filles sélectives lorsqu'elles se retrouvent dans leur équipe (cf. supra). Enfin, elles ne respectent pas toujours les consignes scolaires, par exemple en ce qui concerne les tenues portées lors du sport à l'école : 
Aujourd'hui, Hélène est en jupe et en bottes pour la séance de hockey. Sur le bord du terrain, elle encourage Mélanie, qui joue avec son équipe et dit : «elle n'a toujours pas eu la balle (le palet)! ». (journal de terrain du 14 novembre 2011)

Enfin, leur enseignant considère que certaines d'entre elles éprouvent de réelles difficultés scolaires ; deux de ses membres ont déjà redoublé.

Au-delà de ces deux grands groupes, viennent ensuite des duos relativement autonomes (Déborah et Suzana; Lois et Charlotte). Suzana et Déborah passent leur temps libre à discuter, notamment de la passion pour le cheval de Déborah ou de leur univers domestique (relations de fratrie, parentales, ou avec des animaux) qu'elles partagent aussi aux adultes qui les entourent (animateurs, enquêtrice), ce qui peut paraître contraire au processus d'autonomisation marquant l'entrée dans l'adolescence (De Singly, 2006). Elles observent aussi les jeux des camarades et les côtoient souvent individuellement : groupe de Justine, certains garçons (Mathurin) ou élèves plus jeunes avec qui Déborah partagent certains jeux enfantins tandis que Suzana apprécie des jouets associés à l'enfance :

Sur autorisation de leur enseignant, Lois, Justine, Juliette, Charlotte, Sandrine et Lucile rentrent en classe pour la récréation et dessinent sur leurs cahiers modèles (de stylisme). Lois a amené un cahier de coloriage Petshop. Suzana m'apprend qu'elle a des Petshop chez elle et qu'elle y joue encore parfois (comme Alice). (journal de terrain du 18 novembre 2011)

Dans le même temps, Suzana, qui a une grande soeur, apporte des objets associés à l'adolescence (cahiers de mode, magazines sur les stars) qui intéressent ses camarades, y compris le groupe de Justine :

Suzana et Justine ont amené des cahiers de stylisme aujourd'hui pour occuper leur temps libre : certains modèles y sont déjà habillés, d'autres sont nus (mais sans attribut sexuel); les enfants doivent dessiner leurs habits. Charlotte dit qu'elle va en avoir un aussi. Justine distribue des feuilles à Sandrine et Lucile. (Journal de terrain du 7 novembre 2011)

Déborah, aux longs cheveux tressés, porte régulièrement un legging avec un haut en coton à strasses, des tenues sportwear lors des jours de sport (sweat Adidas, legging) mais peu de marques et de tenues « féminines » à la mode. Suzana a des cheveux longs toujours lâchés et est souvent vêtue d'un haut uni avec un personnage (High School, Annah Montana, Hello Kitty, etc.) et d'un pantalon blanc ou noir en coton. Si Déborah se montre engagée en cours d'Éducation Physique et Sportive et riposte à certains garçons (cf. infra), Suzana apprécie peu les activités physiques et sportives : 
Suzana et Alice me racontent qu'elles n'ont pas aimé le cross. Suzana est arrivée 105, Alice 106, Johan 107. Il dit « en plus, on s'était pas entrainés; on a couru $2 \mathrm{~km} 5$ ». (Journal de terrain du 14 novembre 2011).

Issue d'une famille étrangère, Suzana a progressé depuis son arrivé à l'école mais connaît toujours des difficultés avec la langue française. Déborah obtient des résultats satisfaisants selon son enseignant.

Lois et Charlotte constituent un deuxième duo. Proches du groupe de Justine dont elles partagent certaines activités, via Charlotte que les premières apprécient, elles prolongent leurs relations en dehors de l'école par de nombreuses conversations téléphoniques.

Aujourd'hui, Charlotte et Lois ont un cahier de stylisme et des crayons de couleurs mais n'en feront rien de la récréation. Elles s'informent sur la dispute entre Sandrine et Justine. Après s'être absentée quelques instants, Lois demande sèchement à Ophélie et Déborah de laisser son cahier laissée sur le rebord de la fenêtre. Charlotte lui demande si elle souhaite qu'elle l'appelle ce soir (journal du 15 mai 2012)

Charlotte et Justine et al. partagent le goût de l'apparence féminine soignée et discrète (jupe à volants, petit boléro, ballerines noires, bandeau dans les cheveux, chèche aux couleurs pastel, petits anneaux dorés aux oreilles), combinée à des tenues sportwear pour l'Éducation Physique et Sportive, ce qui marque son attachement au respect des consignes scolaires. En revanche, Lois porte des vêtements aux couleurs vives (chaussures façon Converse violettes, collant fushia) et/ou avec de gros motifs (collant léopard, pantalon batik aux couleurs multiples). Elle possède aussi des accessoires que ne portent pas ses camarades de CM2 (grosses boucles d'oreille pendantes, lunettes de soleil blanches sur la tête, etc.). Certaines de ses tenues vestimentaires (comme le port de sandalettes sans attache au talon) complexifient sa participation aux cours d'Éducation Physique et Sportive et Lois présente plus globalement des difficultés d'accès aux jeux sportifs et à répliquer aux provocations de certains garçons qu'elle et Charlotte côtoient. En effet, celle-ci est l'amoureuse de Marc, leader de certains garçons.

A la première séance de balle ovale qui a lieu à l'extérieur, les enfants se répartissent en petits groupes afin de s'exercer à lancer/rattraper le ballon de rugby. Lois lance le ballon en l'air, sans destinataire précis ; il retombe au centre du groupe. Puis les enfants font un match par équipe. Lois dit « ils sont toujours regroupés, ils nous voient jamais (en parlant de son équipe) ». Sur le terrain, elle attend les mains dans les poches puis s'adresse à Suzana: «on n'existe pas Suzana!». Celle-ci réplique « $\mathrm{Tu}$ sais, on a qu'à faire les Pom-pom grils!». Lois répond « $\mathrm{T}$ 'as vu comment ils poussent ! » (journal de terrain du 12 septembre 2011). 
En dehors de leurs nombreuses discussions, Charlotte, accompagnée de Lois, passe aussi certaines récréations à s'occuper de sa petite soeur, scolarisée en CP. Lorsqu'elle se retrouve seule, Lois apprécie les jeux de société partagés avec d'autres élèves (jeu des fantômes, labyrinthe, cartes, etc.). Toutes deux obtiennent des résultats scolaires satisfaisants et ne sont pas décrites comme de bonnes élèves par leur enseignant.

Enfin, une dernière élève, Ophélie, est isolée de sa classe. Lorsque cela est possible, elle partage avec une élève de CM1 (d'origine étrangère et ayant redoublé) des jeux physiques (bousculades, se monter sur le dos, etc.). A défaut, elle observe les activités des filles de CM2 en tentant d'y prendre part.

A la récréation, Mélanie joue à la maîtresse avec d'autres filles en classe : elles s'amusent à se corriger les fautes sur le tableau. Puis elles écrivent les prénoms de Justine, Clémence, Juliette qu'elles associent à un prénom de garçon: « Justine + François » (élève marginalisé l'an passé). Ophélie tente de se joindre à elles mais Mélanie intervient sèchement: «tu dégages, tu n'as rien à faire là !». (Journal de terrain du 4 juin 2012)

Seule élève de CM2 avec un sac à dos à roulettes, habituellement réservé aux élèves plus jeunes, elle est souvent vêtue, quel que soit le calendrier scolaire, d'un jean et pull ou polaire unis avec des baskets (Nike) ou des chaussures en toile façon converse (non pas de marque) dont les lacets sont régulièrement défaits. Elle porte les cheveux mi longs toujours lâchés et sans aucun accessoire féminisant sa tenue. Même si Ophélie apprécie les bousculades en cour de récréation, elle se montre plus à l'aise comme supportrice sur les bords de terrain qu'au sein des jeux sportifs. Elle adopte alors un vocabulaire qui se démarque de celui habituellement utilisé en classe par ses camarades :

En séance de hockey, Ophélie suit les actions du match de deux équipes sur le bord de terrain. Elle dit à un moment donné « Je gueule moi ! ». (journal de terrain du 14 novembre 2011)

Enfin, même si elle n’a jamais redoublé, Ophélie éprouve des difficultés scolaires selon son enseignant.

Comme le graphique 1 l'indique, ces sous-groupes affinitaires entretiennent des relations d'intensité variée. Bien que la plupart de ces filles possèdent un sentiment d'appartenance à leur groupe de sexe, ces éléments montrent que l'affiliation à un groupe affinitaire tend à les différencier, de par des pratiques ludiques, usages du corps, de l'espace de la cour de 
récréation et modes d'interaction spécifiques. Deux conflits impliquant des filles aux groupes affinitaires distincts vont venir illustrer cette dernière dimension.

\section{Le conflit par dépréciation : un mode de réaffirmation de sa domination}

Parmi les conflits récurrents repérés au cours de l'année de CM2, le groupe de Justine s'est opposé à Lois. Cette mésentente, démarrée à partir du départ de Charlotte pour rejoindre Lois, s'est développée sur plusieurs semaines.

Justine et ses amies apprécient Charlotte qu'elles côtoient depuis plusieurs années mais leurs relations sont distantes avec Lois. Alors qu'elle a quitté leur groupe, ce n'est pas Charlotte qui constitue la cible de leurs reproches mais Lois. Justine et al. lui reprochent d'abord son manque de distance vis-à-vis de la relation amoureuse entre Charlotte et Marc ; Lois ne restant pas à leurs yeux, suffisamment à la place qu'elles considèrent être la sienne. Elles l'accusent même d'être amoureuse de Marc. Si la norme de fidélité amicale paraît en jeu, à l'instar de nombreux conflits enfantins (Monnot, 2013), très vite, d'autres arguments sans lien apparent sont mentionnés par Justine et al. pour justifier la mise à l'écart de Lois :

Selon Clémence, Justine, Juliette, Thomas et Lucile avec qui je mange à table, Lois serait aussi amoureuse de Marc, c'est pourquoi elle ne laisserait pas Charlotte et Marc tranquilles. Justine fait ensuite remarquer que son groupe serait «moins chochotte » que Lois : " elle râle et dit qu'elle a mal dès qu'on la touche ». Après manger, toutes se dirigent vers le camp adverse (Charlotte et Lois) au fond de la cour, comme pour les provoquer. Au moment de rentrer en classe, Juliette se plaint auprès de l'enseignant que Lois et Charlotte les ont traitées de «pétasses » et que Romain a poussé Thomas. (Journal de terrain du 26 septembre 2012)

Au manque de tenue de Lois (respect des sentiments de son amie, insulte qui amène Juliette à interpeler son enseignant), s'ajoute son caractère «chochotte » soulevé par Justine, leader de son groupe. Grande et carrée, pratiquant le judo et l'équitation, sa stature ainsi que ses dispositions sportives et agonistiques l'avantagent en cours d'Education Physique et Sportive mais aussi dans ses rapports sociaux, y compris avec les garçons. On peut ainsi supposer que Justine, en utilisant le terme « chochotte », incrimine les dispositions sexuées de Lois, en lui reprochant de ne pas disposer des mêmes ressources physiques qu'elle et de se conduire selon un genderism féminin qu'elle ne cautionne pas. Au contraire de Justine qui répond aux affronts des garçons, Lois pleure et compte sur la solidarité des premières lorsqu'elle est confrontée à la même situation :

Avant de rentrer en classe, Romain, qui s'est fait des pics au gel aujourd'hui, s'amuse à provoquer les filles qui attendent devant le bâtiment. Il a oublié sa fiche d'exposé et demande à Lois de l'accompagner voir la maîtresse pour la prévenir. Elle lui répond que cela ne la concerne pas et ajoute: «Laure (une élève de CM1), t'as qu'à y aller toi ». Il donne alors une tape à Lois qui claque 
fort. Lois lui dit : «Aïe, t'es chiant hein !» mais ne riposte pas, contrairement à Justine, Clémence et Léa qui se mettent immédiatement à poursuivre Romain. Lois a les larmes aux yeux. Clémence va alors chercher Romain et l'entoure de ses bras. En le rapprochant de Lois, elle dit à Romain : « tu t'excuses maintenant ! ». Il se laisse faire. (journal de terrain du 15 mai 2012)

Son comportement de genre est également visé à travers son apparence vestimentaire. C'est Juliette, autre membre influent du groupe, qui critique directement les goûts vestimentaires de Lois. Soucieuse de son apparence physique (cf. supra), Juliette désigne Lois de «fille pyjama » lors d'un échange informel, alors que celle-ci porte un pantalon ample et coloré. Associé à l'univers de l'enfance, ce type de vêtement est déprécié par les collégiens (Mardon, 2010) et dans notre cas, par Justine et al., qui ont visiblement intégré leurs codes de conduite vestimentaire. Enfin, c'est la situation familiale et socio-économique de Lois, dont la mère est au chômage une partie de l'année, qu'évoque Justine.

A la cantine, je mange à la même table que Lucile, Juliette, Clémence, Justine. Juliette me dit qu'elles se sont réconciliées avec Charlotte mais pas du tout avec Lois. Justine m'explique que la maman de Lois est au chômage et pour faire croire qu'elle cherche un emploi, elle fait manger Lois chez Charlotte. (Journal de terrain du 3 octobre 2012).

Si les propos stigmatisants visent fréquemment les individus marqués par la précarité économique (Zotian, 2009), la disqualification de la situation économique familiale de Lois, doublée d'une dépréciation de ses dispositions sexuées (allure vestimentaire et usage du corps) constitue un moyen pour Justine et al. de se relégitimer, dans le contexte où l'un des leurs a choisi de les quitter pour une tierce personne. Les données récoltées montrent que les filles sélectives sont toutes issues des classes moyennes stables et de précédents travaux ont pu insister sur l'importance de la position parentale dans l'adoption de pratiques récréatives (Mennesson, Julhe, 2012). Les filles sélectives pratiquent plusieurs activités sportives en dehors de l'école, notamment des sports de combat ou collectifs, dont le rugby. Dans la région étudiée, ce sport est valorisé, ce qui explique en partie le choix de certaines filles sélectives. Lois quant à elle, est issue des classes moyennes mixtes (père cadre, mère au chômage puis agent de service) et pratique depuis plusieurs années un instrument de musique et la danse, peu propices à l'acquisition de dispositions agonistiques, mobilisables dans les rapports sociaux de sexe par exemple.

Justine, très sportive, considérée comme la première de la classe par son enseignant et ses camarades, joue un rôle central dans la dépréciation de Lois, comme dans le conflit l'opposant à Sandrine (cf. infra). Ces dispositions scolaires et sportives, reconnues par ses pairs, lui permettent d'établir un rapport de force vis-à-vis de Lois ou d'autres camarades. 
Justine jouit en effet d'un capital symbolique certain (Bourdieu, 1994), au principe du classement des élèves. Ces derniers expérimentent ces rapports de force, sans forcément expliquer leur origine, comme le montrent les extraits d'entretien avec Sandrine. Jadis meilleure amie de Justine puis rejetée par cette dernière, elle témoigne de son influence auprès des pairs, comme plus largement de son groupe :

« Et ça t'attriste (de t'être disputée avec Justine)?

Ben un peu parce que bon, après moi je me retrouve toute seule, parce que toutes les autres copines la suivent (à Justine).

Elle a de l'influence Justine?

Oui.

Comme Clémence et Juliette aussi ?

Ben Clémence et Juliette en fait, elles me suivaient, mais Justine les a influencées donc elles sont parties avec elle et depuis elles me font la tête.

Et vous n'avez pas pu en discuter?

Non. Parce que c'est elles qui auront le dernier mot. »

Si l'exclusion d'un membre permet de se valoriser et d'établir une relation de pouvoir (Gayet, 2006), dans le cas présent, c'est la dépréciation sociale et sexuée de Lois qui permet au groupe de Justine de réaffirmer sa position centrale au sein des pairs. Dans d'autres cas, le conflit ne cherche pas tant à disqualifier qu'à défendre son appartenance aux pairs. Dans ce cas, il peut se présenter comme particulièrement virulent.

\section{Le conflit brutal comme moyen de défendre une place parmi les pairs}

Un autre conflit féminin, déclaré dès le début d'année scolaire et maintenu jusqu'à la fin de celle-ci, a concerné Alice, nouvelle élève et Ophélie, une ancienne faiblement intégrée. Si la première est initialement membre du groupe de Mélanie et al., Ophélie se retrouve la plupart du temps seule ou en présence d'une élève plus jeune, également marginalisée. Alors que le conflit opposant Justine et al. et Lois peut être caractérisé de sourd, dans le sens où, la plupart du temps, l'affrontement direct est évité, Alice et Ophélie, qui se définissent comme deux « ennemies », se confrontent en recourant à la violence verbale et physique sans justifier leur différent, contrairement au premières, si ce n'est en se renvoyant les tords :

En route vers la salle de sport depuis la classe, Alice et Ophélie se disputent alors que l'enseignant est loin devant avec d'autres élèves qui portent le matériel. Ophélie me dit qu'elle veut « tuer Alice» car elle lui aurait fait un doigt d'honneur. De son côté Alice affirme qu'Ophélie lui aurait tiré les cheveux et l'aurait étranglé. Pendant la séance de sport scolaire, la dispute se poursuit, Ophélie dit à Alice : «je vais te violer». Les autres élèves qui l'entendent se moquent d'elle, ce qui l'énerve 
d'autant plus. Alice se rapproche de l'enseignant comme pour se protéger. (Journal de terrain du 21 novembre 2011).

Alice et Ophélie s'insultent, puis, en marge des autres conflits féminins observés, Ophélie recourt à la violence physique, à l'instar de certains garçons de la classe. Ce registre d'opposition est d'ailleurs représentatif des manières de faire d'Ophélie. Nous l'avons déjà vu étrangler un élève plus jeune à la sortie de la cantine et il lui arrive de s'emporter sous l'emprise de ses émotions, en criant ou en renversant des chaises, ce que l'on n'observe pas chez les autres filles :

En début de récréation, je discute avec Sandrine, Suzana, Ophélie et Déborah. Je fais remarquer à Ophélie qu'elle a de nouveau ses lacets défaits. C'est Déborah qui les lui refait. Avant de nous rejoindre, elle crachait à deux reprises dans la cour. Un peu plus tard, certains élèves rentrent en classe pour jouer à l'ordinateur. Ophélie les rejoint. A un moment, elle s'énerve, renverse une chaise car d'autres élèves auraient sauté son tour de jeu. Ophélie ajoute «et encore, je ne suis pas vraiment énervée, sinon je bouscule tout, je crie ! ». (journal de terrain du 3 octobre 2011)

Dans ce contexte, le comportement d'Ophélie est davantage assimilé par ses camarades à un genderism masculin. Ces comportements de genre jouent un rôle important dans son intégration relative à sa classe, notamment auprès des garçons qui la rejettent plus explicitement, comme s'ils signifiaient à Ophélie qu'elle ne fait pas non plus partie des leurs.

Sur le chemin de l'algéco pour réaliser un entretien avant le repas à la cantine, Mathurin lance à Ophélie «couilles molles» et Guillaume qui l'accompagne : «regarde, elle a des yeux rouges de vampire » alors que celle-ci ne leur a rien dit. (journal de terrain du 21 novembre 2011)

Le conflit Ophélie/Alice est également marqué par l'intervention d'adultes. Face à une menace de mort transmise sur papier par Ophélie à Alice et les actes de violence entre les deux filles, le directeur de l'école intervient sur demande de la mère d'Alice et impose aux filles une « distance de sécurité » de deux mètres.

A la reprise du temps scolaire après la pause méridienne, Ophélie arrive de chez elle en tirant un cartable à roulettes lumineuses. Aussitôt son sac posé, elle sort un cahier de brouillon avec un stylo. Devant la classe où attendent d'autres élèves, elle se met à chanter plusieurs fois la chanson Sacré Charlemagne de France Gall. Alice s'approche pour regarder les paroles. Ophélie lui fait remarquer qu'elle n'est pas censée s'approcher d'elle : «à 2 mètres » lui dit-elle. (Journal de terrain du 17 janvier 2012)

Transgressant les frontières générationnelles (Zotian, 2009), l'intervention d'adultes n'adoucit par la mésentente entre les deux filles. Au contraire, elle se poursuit autour de la 
figure de Judith, élève de CM1 que côtoie régulièrement Ophélie dans la cour de récréation. En fin d'année scolaire, elle constitue pour Alice une camarade de jeu de repli lors de son rejet du groupe de Mélanie et al..

Avant le repas, Ophélie passe à côté d'Alice et la bouscule. Cette dernière s'écarte en disant «j'en ai marre! ». Après la cantine, Alice cherche Ophélie pour savoir pourquoi elle l'a bousculée. Quand elle s'approche d'Ophélie, celle-ci lui dit « dégage !». Alice recule en disant « tu vois! » à Déborah. Plus tard, au cours de la récréation, j'aperçois Ophélie qui lance toute seule des turbulots, assise sur une marche des algécos, non loin d'Alice et Judith ; je lui demande ce qui lui arrive. D’un air énervé, elle répond « je me fais chier! ». Elle me raconte qu'elle n'est plus copine avec Judith qui « traîne » désormais avec Alice. Alice se lève et Judith la suit. (Journal de terrain du 14 mai 2012)

Par une bousculade, Ophélie exprime à sa rivale le fait qu'elle ait dépassé les bornes en la privant de sa seule camarde de jeu à l'école. Notons que toutes deux fréquentent peu d'enfants en dehors de l'école. En ce sens, le maintien d'une relation sociale dans la cour de récréation constitue un enjeu central. Plus qu'une relation d'amitié entre Ophélie et Judith ou cette dernière et Alice, leurs contacts en cour de recréation permettent à chacune de ne pas se retrouver seules, de rompre avec l'isolement et ainsi conserver un lien avec la communauté enfantine. Si la confiscation d'une amie pour le groupe de Justine et al., remettait en question sa supériorité, pour Ophélie et Alice, toutes deux rejetées par leurs pairs, la privation d'une relation interpersonnelle questionne leur identification à leur classe d'âge et, pour Ophélie, sexuée.

Ainsi, au cours de ce conflit inscrit dans la durée, tout se passe comme si Ophélie et Alice, dont la position est semblable au sein du groupe des pairs, se disputaient de manière violente une place parmi celui-ci. Toutes deux font en effet l'objet de stigmatisations de la part d'autres élèves, l'une sur son comportement, l'autre davantage sur son apparence corporelle, contrainte par sa situation familiale précaire. Alice vit depuis peu dans la commune avec sa mère et sa petite sœur dans un logement social. Sa mère, qui a obtenu un statut d'handicapée, ne travaille pas et seules les aides sociales contribuent aux ressources familiales. Dans ce contexte, Alice se plaint auprès de sa mère de sa condition vestimentaire, importante dans la légitimité des filles :

Lorsque j'arrive dans la cour de récréation, Alice se précipite vers moi et me demande si j'ai le numéro de sa mère. Elle est embêtée car elle a une fermeture de sa chaussure cassée et celle-ci tient mal sur son pied. Je lui dis de voir cela avec le directeur. Puis, quelques minutes après, elle revient en se blottissant contre moi : «il m'énerve !», en parlant du directeur, qui n'a pas voulu appeler chez elle. (journal de terrain du 5 juin 2012) 
Toutes deux sont issues de milieux populaires mais Alice connait une situation familiale plus précaire qu'Ophélie. Cette précarité complexifie aussi sa participation à des activités de loisirs extra scolaires auxquelles elle n'a jamais été inscrite, ce qui n'est pas le cas d'Ophélie qui fait du karaté pour se « défouler », après une année de judo. Ces dispositions agonistiques renforcent sa domination sur Alice dès qu'elle mobilise le registre physique. Alice a en effet peur de prendre des coups de la part d'Ophélie et sollicite la protection des adultes (cf. supra). Leur manière de faire montre également que les conflits semblent d'autant plus vifs et tenaces qu'ils concernent des filles à la position reléguée au sein du groupe des pairs, pour lesquelles l'enjeu d'appartenance aux pairs est capital.

\section{Conclusion :}

S'intéresser aux interactions au sein d'un seul groupe de sexe dans la cour de récréation, permet de prendre au sérieux le rôle des liens affinitaires dans le processus de socialisation des enfants. Ces liens, qui se matérialisent dans des sous-groupes affinitaires, favorisent certaines pratiques récréatives et modes d'interaction sociale et font l'objet de tensions. La (re)définition des liens affinitaire et les conflits qui les accompagnent contribuent au positionnement des filles au sein d'un groupe de sexe et de la classe. Pour cela, elles s'appuient sur des différences sociales qui se construisent à l'école - notamment au sein des groupes de pairs - mais aussi en dehors, au sein de la classe, dans les familles ou lors d'activités extra scolaires.

Les deux conflits pris pour exemple montrent que les filles, en fonction de leur groupe affinitaire et ressources, gèrent de manière différente leur antagonisme. D'un côté, Justine et al., sportives, en réussite scolaire et issues des classes moyennes, recourent à des formes sourdes - éviction et disqualification indirecte - pour réaffirmer leur supériorité sur Lois, et plus largement sur l'ensemble des filles. Pour cela, elles mobilisent des justifications liées à la position sociale parentale et surtout aux dispositions sexuées de Lois, objectivables dans son allure vestimentaire, ses pratiques récréatives et certaines situations comme les jeux d'opposition. Au contraire, chez Ophélie et dans une moindre mesure Alice, toutes deux issues des classes populaires, l'enjeu ultime d'identification au groupe des pairs justifie le recours aux insultes et à la violence physique, à défaut d'une disqualification explicite. Ces deux cas montrent également que les conflits durables concernent plus particulièrement des élèves à la position proche au sein des pairs ; les élèves plus fortement différenciés n'ont pas besoin de faire remarquer au quotidien leur différence et tendent à s'ignorer, à l'instar de ce 
qu'avaient montré W. Lignier et J. Pagis (2012) au cours de leur enquête sur les procédures de classements des métiers par des élèves.

Ce travail illustre aussi comment les dispositions sexuées, en partie façonnées par la socialisation sportive elle-même influencée par l'origine sociale, peuvent plus ou moins constituer une ressource pour les enfants au sein de leurs rapports sociaux. Si la popularité est multifactorielle (Adler, Kless, Adler, 1992), ce travail insiste sur le poids des dispositions agonistiques dans le contexte étudié, facilitées par la pratique de certains sports (de combat ou collectifs) dans l'instauration de rapports de domination, y compris chez les filles. Il permet d'enrichir la connaissance des rapports sociaux enfantins, en insistant sur la place qu'y joue le corps sur les sociabilités féminines, celui-ci constituant un «pense bête » du social (Bourdieu, 1980).

Enfin, les résultats mettent au jour une certaine homologie entre positions parentales et enfantines via les groupements affinitaires différenciés et hiérarchisés. Cela confirme que les rapports enfantins sont modelés à l'image des rapports inégaux qui organisent la société adulte (Geay, 2000) et marqués par l'intériorisation précoce du sens de «l'ordre social » (Darmon, 2001). Ce travail laisse à penser que les groupes affinitaires constituent une instance de socialisation tendant à renforcer les dispositions des enfants acquises dans leur famille et lors de loisirs extra scolaires même si leurs membres ne sont pas strictement identiques. Ces premiers éléments invitent à prendre davantage en compte l'articulation entre sphères scolaire, familiale, des loisirs et des pairs dans l'étude des relations sociales qui se développent au sein de l'école et à la manière dont les groupes affinitaires participent à la construction de rapports différenciés aux autres, aux adultes et aux normes scolaires.

\section{Bibliographie :}

- ADLER P., KLESS S.-J., ADLER P. 1992, « Socialization to Gender Roles : Popularity among Elementary School Boys and Girls », Sociology of éducation, vol. 65, n³, p.169-187. - AUGUSTINS G. 1988. «Le jeu de billes. Lieu de la raison, lieu de la passion », Ethnologie française, 1, janvier-mars, tome 18, pp. 5-14.

- BOURDIEU P. 1980. Le sens pratique, Paris, Éditions de Minuit.

- BOURDIEU P. 1994, Raisons pratiques sur la théorie de l'action, Paris, Éditions du Seuil.

- BROUGERE G. 1999, Les expériences ludiques des filles et des garçons, in Y. Lemel, B. Rouet (dirs), Filles et garçons jusqu'à l'adolescence. Socialisations différentielles, Paris, L'Harmattan, p. 199-222. 
- CARRA C. 2008, «Violences à l'école élémentaire. Une expérience enfantine répandue participant à la définition du rapport aux pairs », L'Année sociologique, vol. 58, p. 319-337.

- COMORETTO G. 2010, «La cantine », lieu privilégié de construction de la sociabilité enfantine », Actes du colloque international Alimentation, cultures enfantines et éducation, Centre Européen des produits de l'enfant, Poitiers, Université de Poitiers.

- DARMON M. 2001, « La socialisation, entre famille et école. Observation d'une classe de première année de maternelle », Sociétés \& Représentations, ${ }^{\circ} 11$, p.515-538.

- DARMON M. 2008, Devenir anorexique. Une approche sociologique, Paris, La découverte.

- DELALANDE J. 2001, La cour de récréation, contribution à une anthropologie de l'enfance, Rennes, Presses Universitaires de Rennes.

- DÉTREZ, C. 2014, Sociologie de la culture, Paris, Armand Colin.

- DE SINGLY F. 2006. Les adonaissants, Paris, Armand Colin.

- GAYET D. 2006, «L'univers social des petits », Les sciences de l'éducation - pour l'Ere nouvelle, vol. 39, p. 53-67.

- GEAY B. 2000, « Processus de socialisation, groupe de pairs et cultures enfantines », In D. Saadi-Mokrane, Sociétés et cultures enfantines, Lille, Presse Universitaire de Lille 3.

- GEAY B. 2003, Engagement dans les activités ludiques et appropriation différentielle des normes de compétition et de coopération à la fin de l'école primaire, colloque Normes sociales et processus cognitifs, Poitiers, MSHM, juin.

- LIGNIER W., al. 2012, « La différenciation sociale des enfants », Politix, n 99, p.9.

- LIGNIER W., PAGIS J. 2012, «Quand les enfants parlent l'ordre social. Enquête sur les classements et jugements enfantins », Politix, $\mathrm{n}^{\circ}$ 99, p. 23-49.

- LIGNIER W., PAGIS J. 2014, «Inimitiés enfantines. L'expression précoce des distances sociales », Genèses, n 96, p. 35-61.

- MARDON A. 2010, «Sociabilités et travail de l'apparence au collège », Ethnologie française, vol. $40, \mathrm{n}^{\circ} 1$, p. 39-48.

- MONNOT C. 2013, Petites filles. L'apprentissage de la féminité, Paris, Editions Autrement, $1^{\text {ère }} \mathrm{Ed}, 2009$.

- MENNESSON C., JULHE S. 2012, «L'art (tout) contre le sport ? », Politix, n99, p. 109128.

- OCTOBRE S. 2003, «Les 6-14 ans et les équipements culturels. Des pratiques encadrées à la construction des goûts », Ministère de la Culture et de la Communication, Département des Etudes et de la Prospective, Revue de l'OFCE, n86, p. 143-154. 
- OCTOBRE S., JAUNEAU Y. 2008 ; «Tels parents, tels enfants ? Une approche de la transmission culturelle », Revue française de sociologie, vol. 49, n²4, p. 695-722.

- RAYOU, P. 1999, La grande école. Approche sociologique des compétences enfantines, Paris, PUF.

- ROUCOUS N. 2007, « Les loisirs de l'enfant ou le défi de l'éducation informelle », Revue française de pédagogie, $\mathrm{n}^{\circ} 160$, p. 63-73.

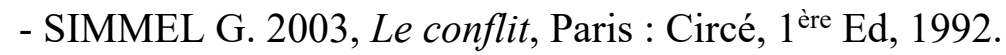

- SIROTA R. 1988, L'école primaire au quotidien, Paris : Presses Universitaires de France.

- ZOTIAN E. 2009, Grandir à Belsunce, les catégories ordinaires de l'expérience enfantine dans un quartier de Marseille, Thèse de doctorat en sociologie, Marseille, EHESS.

${ }^{i}$ Cet article s'inscrit dans le cadre d'un programme de recherche financé par l'Agence Nationale de la Recherche sur le rôle de la socialisation corporelle dans la construction du genre des enfants (Normenfan, Mennesson, Neyrand, dirs). 